\title{
Practice guidelines for peer support among educators during a curriculum innovation
}

\author{
M Shawa, BSc Nursing, MPH; Y Botma, PhD \\ School of Nursing, Faculty of Health Sciences, University of the Free State, Bloemfontein, South Africa
}

Corresponding author: M Shawa (mirriamshawa06@gmail.com)

Background. Curriculum transformation in nursing education addresses changing healthcare needs of communities. However, without ongoing support of educators, the fidelity of curriculum enactment could be compromised. Nursing education institutions in Lesotho implemented a competencybased curriculum that required novel pedagogical approaches. New facilitation approaches can challenge implementers, as was observed during the implementation of a new curriculum for the midwifery programme in Lesotho. Without ongoing faculty development and support, the educators resorted to supporting one another. However, the sustainability and effectiveness of the unstructured peer support could be compromised; hence the need to develop guidelines to enhance peer support among educators during curriculum innovation.

Objective. To develop and validate guidelines to enhance peer support among educators during curriculum innovation.

Methods. A qualitative research design with multiple data collection methods was conducted, guided by the World Health Organization Handbook for Guideline Development as the framework. Three interrelated phases, inclusive of an integrative review, an exploratory qualitative study, guideline development and validation, were conducted. External reviewers validated the developed guidelines by means of a Delphi survey.

Results. Five priority areas were identified for the practice guidelines, i.e. attributes of peer supporters, peer support strategies, content/support needs, outcomes of peer support, and monitoring and evaluation of the peer support strategy. Recommendations were formulated for each priority area.

Conclusion. These practice guidelines provide relevant recommendations that can enhance peer support among educators in nursing education programmes during curriculum innovation. The recommendations serve as a blueprint and provide direction for the structured peer support engagements.

Afr J Health Professions Educ 2020;12(4):186-190. https://doi.org/10.7196/AJHPE.2020.v12i4.1388

Curriculum transformation in higher education institutions contributes to enhancing the quality of graduates and prepares them to address emerging socioeconomic and health challenges in different communities. ${ }^{[1,2]}$ The successful execution of a transformed curriculum depends on the capability of the faculty as the drivers of curriculum implementation. ${ }^{[3]}$ However, transforming the curriculum from one learning theory to another that is underpinned by different principles and pedagogical approaches can challenge the educators' existing skill set. Ill-equipped faculty may struggle to implement the curriculum as intended, thereby necessitating support strategies to enhance their abilities to appropriately enact the transformed curriculum. Therefore, educational institutions embarking on curriculum transformation need to proactively formulate clear strategies for relevant ongoing faculty development to support the change process. ${ }^{[4]}$ However, planning and undertaking formal faculty development and capacitybuilding interventions in low- and middle-income countries (LMICs) may be deterred by limited resources. Therefore, LMICs embarking on curriculum transformation may benefit from affordable support strategies such as peer support.

Evidence shows that peer support can sustain and improve the outcomes of an innovation. ${ }^{[5-7]}$ Peers can support one another through encouragement and by providing emotional support and information to improve knowledge and skills. Such support activities and exemplar behaviour may increase peers' self-efficacy and enhance the implementation of the change process. Bandura $^{[8]}$ highlights vicarious experience and verbal persuasion as some of the means by which peers can support one another. Peer support activities could be compromised by the absence of a structured approach. The researchers argue that guidelines for peer support can give direction and enhance the interactions of peers during the change process, as with curriculum innovation. This article describes the guidelines for peer support developed for educators engaged in curriculum change in nursing education in Lesotho, a low-income country in southern Africa.

The context of this study is the implementation of curriculum innovation in the midwifery programme in Lesotho. In 2014, nursing education institutions in Lesotho implemented the first competency-based curriculum (CBC) in the 1-year midwifery programme. The transformed curriculum required a new skill set among nurse educators, who were at different levels of readiness. However, the institutions had no deliberate plan for ongoing support or faculty development. Naturally, the early adopters of the new curriculum provided unstructured support to their peers. Although the unstructured peer support during the midwifery $\mathrm{CBC}$ was successful, there were some limitations, such as lack of administrative commitment, lack of accountability and lack of monitoring and evaluation (M\&E). ${ }^{[9]}$

\section{Methods}

The practice guidelines were developed through a qualitative research design using multiple data collection methods, guided by the World Health Organization (WHO) Handbook for Guideline Development as a framework. ${ }^{[10,11]}$ The guideline development process entailed three separate phases, each addressing a specific objective(s) through interrelated studies (Fig. 1). The study conducted in phase I synthesised existing peer support strategies 
that enhanced the implementation of an innovation or new programme among professionals between 2000 and 2016 through an integrative review. The details and findings of the integrative review have been reported elsewhere. ${ }^{[11]}$ Phase II entailed a qualitative study that described the experiences of midwife educators regarding peer support during the implementation of a new curriculum in Lesotho. Data were collected from 12 midwife educators through unstructured interviews, which were recorded, transcribed verbatim and analysed inductively. The details and findings of this study are reported elsewhere. ${ }^{[12]}$

The third and final phase involved triangulation of evidence from the studies of the two preceding phases, followed by development and validation of the guidelines for peer support. In line with the WHO Handbook for Guideline Development, ${ }^{[11]}$ the researcher established a task team consisting of a methodology expert and curriculum specialist, a

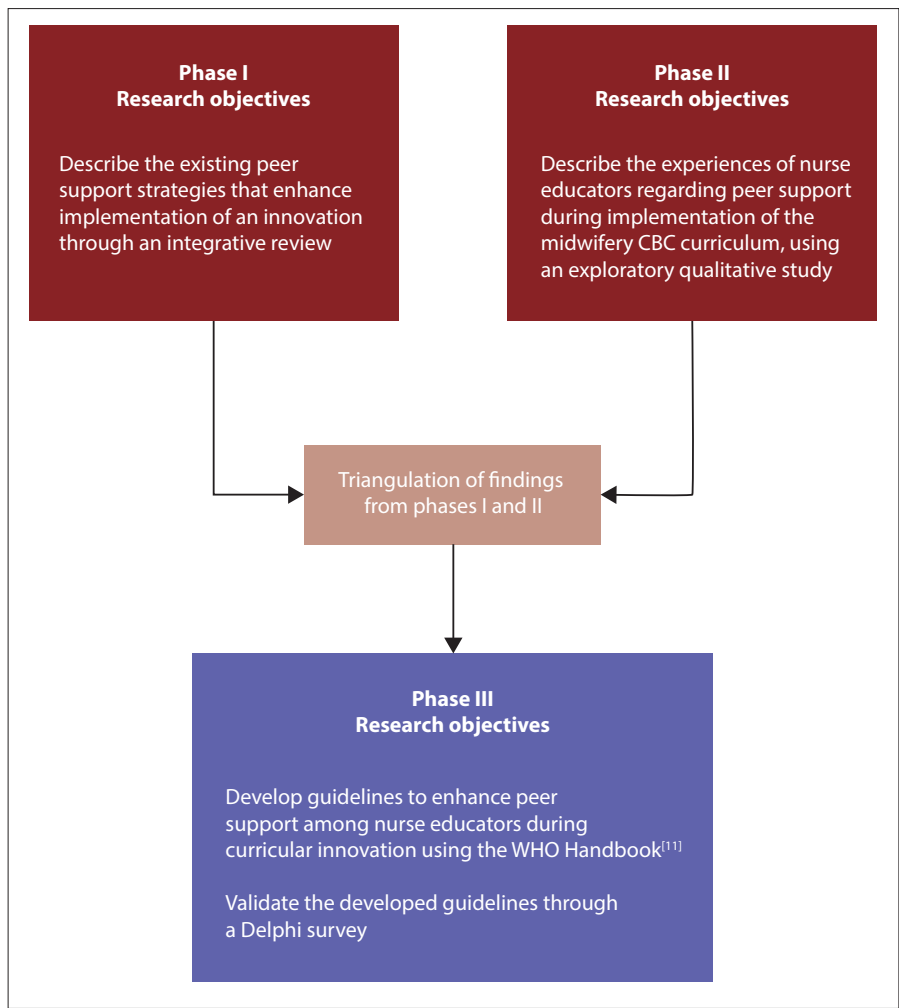

Fig. 1. Methodological process for guideline development. ( $C B C=$ competencybased curriculum; $W H O=$ World Health Organization. ) faculty development expert knowledgeable regarding peer support and the researcher. Based on the triangulated evidence from the studies of the preceding two phases, the task team identified, discussed and agreed on priority areas and recommendations through consensus. Secret voting was used to reach a decision whenever there was a disagreement. Five priority areas and seven recommendations were formulated and evaluated against the quality assessment framework described in the Handbook for Guideline Development. ${ }^{[1]}$ Validation of the guidelines was conducted by a panel of external reviewers through a Delphi survey. A detailed description of the development process and the guidelines are presented in the supplementary material (http://ajhpe.org.za/public/files/1388.pdf).

\section{Rigour of the guideline development process}

Validation by an expert panel contributed to the rigour of the development of the guidelines. The task team purposefully identified 16 experts in nursing education and mentorship from Africa and Asia, based on their qualifications, expertise and experience. Nine reviewers accepted the invitation to participate in the Delphi survey. The expert panel used the 23-item Appraisal of Guideline for Research and Evaluation (AGREE II) tool to evaluate the guidelines through a two-cycle Delphi survey. ${ }^{[13,14]}$ The AGREE II tool addresses 6 domains:

- scope and purpose

- stakeholder involvement

- rigour of development

- clarity and presentation

- applicability

- editorial independence.

The response rates during rounds I and II of the Delphi survey were $89 \%$ and $75 \%$, respectively. Hasson and McKenna, ${ }^{[14]}$ citing Sumsion, suggest that a response rate of $70 \%$ is rigorous for a Delphi survey. The responses from the expert reviewers were analysed using the proportions of agreement for each of the items on the AGREE II tool. The task team made amendments and consolidated the recommendations based on the analyses of both rounds of the Delphi survey. Fig. 2 summarises the guideline validation process.

\section{Ethical approval}

Ethical approval was obtained from the Health Sciences Research Ethics Committee, University of the Free State (ref. no. HSREC 28/2017) and the Lesotho Ministry of Health Research and Ethics Committee (ref. no.

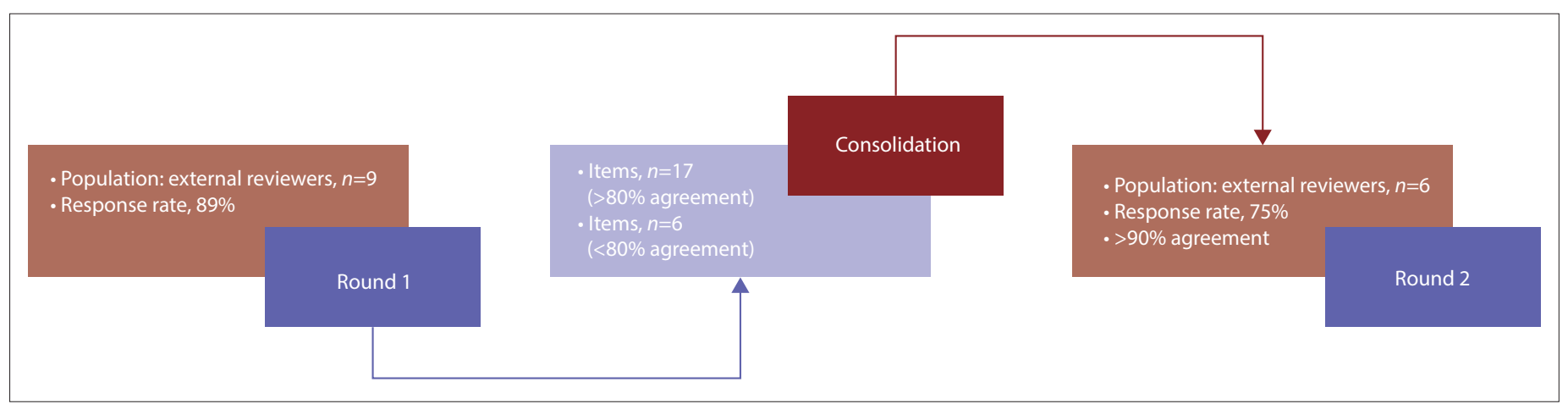

Fig. 2. Summary of the guideline validation process. 
ID 91-2017). All participants in the qualitative study and the Delphi survey received detailed information and participated voluntarily. The external reviewers remained anonymous to one another throughout the validation process. ${ }^{[14]}$

\section{Results}

Five priority areas and seven recommendations were developed for the peer support guidelines. The priority areas are as follows

\section{Priority area 1: Attributes of peer supporters}

This priority area focuses on the qualifications, capabilities and qualities of peer supporters. Evidence suggests that peer supporters require a higher qualification and expertise in a specific discipline, such as a Master's or doctoral degree in nursing/health professions education. However, it is unlikely that many educators in LMICs have the necessary higher qualifications. Therefore, a formal qualification in nursing/health professions education is acceptable for a peer supporter. Attributes such as experience, motivation and commitment to peer support are valued and readily accepted among peers.

\section{Priority area 2: Peer support strategies}

This priority area focuses on the strategies for providing support and the characteristics of an effective support strategy. Evidence shows that relevant and tailor-made strategies and platforms have positive outcomes and are acceptable and valued by peers receiving support. Strategies include group support approaches and paired techniques. Acceptability and feasibility are high when there is institutional commitment to the strategy.

\section{Priority area 3: Content/support needs}

Tailor-made content is valued and acceptable, and has a positive effect on peers. Assessment to determine the content or support should be done in collaboration with those who need support. The content should be aligned to the new curriculum implementation needs of individuals.

\section{Priority area 4: Outcomes of peer support}

The goals and objectives of the peer support strategy should be directed towards sustaining curriculum innovation, improved curriculum implementation and promotion of professional and personal growth. The commitment of institutional administrators enhances accountability regarding the outcomes of peer support, promotes success of peer support and ultimately sustains curriculum innovation.

\section{Priority area 5: Monitoring and evaluation of the peer support strategy}

M\&E is an essential component of successful peer support, and enhances and sustains peer support strategies. A peer support strategy should have an M\&E mechanism that provides opportunity for feedback and enhances effectiveness of the strategy.

Table 1 presents a summary of the recommendations.

\section{Discussion}

Practice guidelines can enhance peer support interactions among implementers of a transformed curriculum, particularly in resourcelimited institutions that cannot afford ongoing faculty development. The lack of ongoing faculty development and support could compromise the fidelity of the implementation of the transformed curriculum and

Table 1. Summary of guideline recommendations on peer support

Priority area
A1: Attributes of peer supporters

Recommendations

Al: Attributes of peer supporters

A1.1: Peer supporters should be in possession of a formal qualification in nursing/health professions education, be

knowledgeable about the principles guiding the curriculum innovation, experienced in mentoring, motivated and committed to provide support and facilitate the professional growth of the peers

Level of evidence used: moderate

B1: Peer support strategies B1.1: Peer supporters should consider the needs of the peers related to the implementation of curriculum innovation, such as developing appropriate facilitation materials and using relevant pedagogical and assessment methods. Peer supporters should select the most appropriate strategies and platforms to provide support

Level of evidence used: moderate

B1.2: Institutional leadership should ensure that the support strategy has clear goals and objectives, explicit systems and mechanisms to enhance and sustain the effective implementation of the strategy during curriculum innovation Level of evidence used: moderate

C1: Content/support needs C1.1: Peer supporters should collaborate with the peers/faculty to assess and identify support needs to enable the development of relevant and applicable content that is aligned with the implementation of the new curriculum Level of evidence used: moderate

D1: Outcomes of peer support D1.1: Goals and objectives of peer support activities should be aligned with the identified needs and directed towards sustaining the curriculum innovation, capacity building, professional growth, community of practice and scholarship Level of evidence used: moderate

D1.2: Institutions should recognise support strategies as a valued service and commit by allocating resources to meet the departmental/support needs to enhance peer support during a curriculum innovation

Level of evidence used: moderate

E1:M\&E of the peer support strategy E1.1: Institutional leadership should ensure that there is a mechanism for M\&E of peer support strategies used during curriculum innovation

Level of evidence used: moderate 
create an environment for curriculum drift. ${ }^{[15]}$ Botma $^{[16,17]}$ reiterates that educators who are not familiar with the principles underpinning the new curriculum could facilitate curriculum drift. Therefore, without ongoing support for educators during curriculum transformation, curriculum drift is unavoidable. These practice guidelines are contextualised and recommend strategies and processes essential for effective peer support among educators engaged in the enactment of curriculum innovation. Various factors, including qualifications, experience and commitment of support providers, influence the effectiveness of peer support strategies. ${ }^{[5,6,18]}$ However, in LMICs, such as Lesotho, it may not be feasible for most nursing education institutions to have educators with qualifications that are higher than the basic nursing degree. In the absence of highly qualified peer supporters, institutions can utilise knowledgeable and experienced individuals, such as early adopters ${ }^{[17]}$ Institutional leaders also need to develop deliberate faculty plans directed towards building of capacity of potential supporters. ${ }^{[17,19]}$ Peer supporters should also possess effective interpersonal and communication skills to facilitate a positive and collegial environment and interactions during support activities. ${ }^{[5,20,21]}$

The content for the peer support strategy should be well planned and relevant to the curriculum implementation needs of peers. Klinge ${ }^{[22]}$ agrees with Pololi and colleagues that learning occurs naturally when adult students perceive it as relevant to improve their self-efficacy. Ensuring relevant content requires collaborative assessment and identification of support needs. ${ }^{[5,23]}$ The designed content should be administered using appropriate strategies, such as workshops, presentations, meetings, supportive peer reviews and hands-on methods. Role modelling and encouragement further enhance the self-efficacy of colleagues during the change process. ${ }^{[8]}$ The participants may value and prefer engaging and hands-on strategies that are in line with the challenges they are facing. Knowles' work cited by Klinge ${ }^{[22]}$ alludes to the principles of adult learning and emphasises the importance of designing needs-driven support strategies that promote active learning. However, peer support providers in LMICs need to be cognisant of limitations associated with some strategies and platforms, such as connectivity, systems failure and the technological abilities of individuals, ${ }^{[20]}$ which might influence the effectiveness and success of the support strategy.

Outcomes of an effective peer support strategy include sustained curriculum innovation, personal and professional growth and development of a community of practice. ${ }^{[6,7]}$ Peer support approaches that promote self-directedness and critical thinking ought to be encouraged. Besides these positive outcomes, unintended effects, such as negative emotional reactions, might also be experienced and compromise the effectiveness of the support. ${ }^{[5,18]}$ Therefore, establishment of a committee instead of one person working on peer support interventions may create a buffer for the potential emotional strains that individuals may experience. ${ }^{[6,18]}$ Some factors that can compromise the effectiveness of a peer support strategy include disconnections in relationships, power differences, unclear mentoring roles and lack of $\mathrm{M} \& \mathrm{E} \cdot{ }^{[5,18,21]}$ However, critical to the attainment of positive outcomes is the commitment of institutional leadership to the strategy of peer support.

The guidelines allude to the commitment of institutional leadership, which is essential in creating an environment conducive to successful peer support strategies. ${ }^{[4]}$ Such commitment is key to the success of peer support and influences the allocation of resources, accountability and M\&E of the strategy ${ }^{[6,18,21]}$ Both the integrative review and the qualitative study highlighted the importance of administrative endorsement. ${ }^{[9,12]}$ Although $M \& E$ is essential for any effective intervention, these quality assurance mechanisms are sometimes disregarded, leading to delayed identification of challenges and weakness, and subsequently no corrective measures are undertaken. ${ }^{[7,24]} \mathrm{M} \& \mathrm{E}$ can be the mainstay of the support strategy and for sustaining the implementation of curriculum innovation. ${ }^{[7,18]}$

These peer support guidelines can be adapted to different contexts and used among educators in institutions undergoing curriculum transformation in LMICs. Peer support is one of the affordable approaches that can benefit educators in resource-limited institutions.

\section{Conclusions}

The practice guidelines presented in this article are aimed at enhancing peer support engagement among educators in nursing education programmes during curriculum transformation. These contextualised guidelines recommend strategies and processes that address critical aspects of peer support, including attributes of peer supporters, peer support strategies, content/support needs, outcomes of peer support, and monitoring and evaluation of the peer support strategy. The formulated recommendations can serve as a blueprint that gives direction to structured peer support interactions among educators during curriculum innovation in resourcescarce countries. However, these guidelines are not a panacea for all the challenges associated with curricular transformation processes, but form part of the solutions. Therefore, the guidelines should be used together with other strategies that enhance fidelity of curriculum implementation.

Further research is recommended to evaluate the usability and effectiveness of the guidelines in the different institutions that may use them. There is also a need to evaluate the efficacy of the implementation of curriculum reforms funded by the Nursing Education Partnership Initiative (NEPI) in African countries.

Declaration. The study was conducted in partial fulfilment of the requirements for MS's $\mathrm{PhD}$ degree at the University of the Free State, Bloemfontein.

Acknowledgements. The authors acknowledge Ms J Viljoen and Ms E Heyns for language and technical editing of the manuscript, respectively. The external reviewers are acknowledged for their role in validating the developed practice guidelines.

Author contributions. MS and YB conceptualised the study design, MS collected and analysed the data. MS and YB triangulated evidence, developed and consolidated the peer support practice guidelines. MS drafted the manuscript and YB provided critical guidance throughout the process and approved the final manuscript.

Funding. None.

Conflicts of interest. None.

\footnotetext{
. Frenk J, Chen L, Bhutta ZA, et al. Health professionals for a new century: Transforming education to strengthen health systems in an interdependent world. Lancet 2010;376(9756):1923-1958. https://doi.org/10.1016/s01406736(10)61854-5

2. Niehaus E, Williams L. Faculty transformation in curriculum transformation: The role of faculty developmen in campus internationalization. Innov High Educ 2016;41:59-74. https://doi.org/10.1007/s10755-015-9334-7 Snyman S. Reconceptualising of Health Professions Education in South Africa. Pretoria: ASSAf, 2018.

4. Galea S, Fried LP, Walker JR, Rudenstine S, Glover JW, Begg MD. Developing the new Columbia core curriculum A case study in managing radical curriculum change. Am J Public Health 2015;105(Suppl 1):S17-S21. https://dol org/10.2105/ajph.2014.302470

5. Bryant AL, Aizer Brody A, Perez A, et al. Development and implementation of a peer mentoring program for early career gerontological faculty. J Nurs Scholar 2015;47(3):258-266. https://doi.org/10.1111\%2Fjnu.12135
} 
6. Fleming GM, Simmons JH, Xu M, et al. A facilitated peer mentoring program for junior faculty to promote professional development and peer networking. Acad Med 2015;90(6):819-826. https://doi.org/10.1097/ acm. 0000000000000705

7. Magers TL. An EBP mentor and unit-based EBP team: A strategy for successful implementation of a practic change to reduce catheter-associated urinary tract infections. Worldviews Evidence-Based Nurs 2014;11(5):341-343. https://doi.org/10.1111/wvn.12056

8. Bandura A. Human agency in social cognitive theory. Am Psychol 1989;44(9):1175-1184. https://doi. org/10.1037/0003-066x.44.9.1175

9. Shawa M, Botma Y. Peer Support Strategies that Enhance Implementation of an Innovation among Professionals: An Integrative Review. Bloemfontein: University of the Free State, 2020.

10. Harrell MC, Bradley M. Data collection methods: Semi structured interviews and focus groups. Geograph Anal 2009;31:1-147.

11. World Health Organization. Handbook for Guideline Development. 2nd ed. Geneva: WHO, 2014:1-179.

12. Shawa M, Botma Y. Peer Support During Implementation of a New Curriculum: Experiences of Nurse Educators. Bloemfontein: University of the Free State, 2020.

3. AGREE Next Steps Consortium. The Agree II Instrument. 2017. http://www.agreetrust.org (accessed 18 November 2018). 14. Hasson F, McKenna HP. Research guidelines for the Delphi survey technique. J Adv Nurs 2000;32(4):1008-1015. https://doi.org/10.1046/j.1365-2648.2000.t01-1-01567.x

15. Burgess T, Cilliers F. A framework for ethical educational research: Principles and application. 2017. www. healthedu.uct.ac.za/framework (accessed 16 June 2017).

16. Botma Y. Implications of accreditation criteria when transforming a traditional nursing curriculum to a competency-based curriculum Int I Africa Nurs Sci 2014:1(12):23-28. https//doi org/10.1016/jijans 2014.06.002 Botma Y How a monster became a princess: Curriculum development S Afr J High Educ 2014:28(6):1876-1893.
18. Sexton JM, Lord JA, Brenner CJ, Curry CE, Shyn SI, Cowley DS. Peer mentoring process for psychiatr curriculum revision: Lessons learned from the 'mod squad'. Acad Psychiat 2016;40(3):436-440. https://doi. org/10.1007/s40596-014-0274-9

19. Brownie SM, Docherty C, Al-Yateem N, Gadallah MH, Rossiter R. Developing a national competencybased curriculum for technical nurses in Egypt. East Mediterr Heal J 2018;24(9):922-932. https://doi. org/10.26719/2018.24.9.922

20. Bang E. Hybrid-mentoring programs for beginning elementary science teachers. Int J Educ Math Sci Techno 2013;1(1):1-15. https://doi.org/10.18404/IJEMST.97208

21. Bennett S, Paina L, Ssengooba F, Waswa D, M'Imunya JM. Mentorship in African health research training programs: An exploratory study of Fogarty International Center programs in Kenya and Uganda. Educ Heal Chang Learn Pract 2013;26(3):183-187. https://doi.org/10.4103/1357-6283.12600

22. Klinge CM. A conceptual framework for mentoring in a learning organization. Adult Learn 2015;26(4):160-166 https://doi.org/10.1177\%2F1045159515594154

23. Bennett S, Santy J. A window on our teaching practice: Enhancing individual online teaching quality though Bennett S, Santy . A window on our teaching practice: Enhancing individual online teaching quality though
online peer observation and support. A UK case study. Nurse Educ Pract 2009;9(6):403-406. https://doi. online peer observation and support. A UK case study. Nurse Educ Pract 2009;9(6):403-406. https://doi. org/10.1016/j.nepr.2009.01.019

24. Chukwu CL, Mezieobi DI, Uguwanyi BE, Okpoebo CC. Monitoring and evaluation on effective delivery of socia studies for improved academic performance. Rev Eur Stud 2019;11(1):175. https://doi.org/10.5539/res.v1ln1p175

Accepted 17 August 2020 